\title{
CONFORMAL INVARIANTS OF HYPERBOLIC PLANAR DOMAINS
}

\author{
F.G. AVKHADIEV, R.G. NASIBULLIN, I.K. SHAFIGULLIN
}

\begin{abstract}
We consider planar hyperbolic domains and conformally invariant functionals defined as sharp constants for Hardy type inequalities. We study relationships between these functionals and optimal constants in hyperbolic isoperimetric inequalities. The studied Hardy type inequalities involve weight functions depending on a hyperbolic radius of a domain and are conformally invariant. We prove that the positivity of Hardy constants is connected with existence of some hyperbolic isoperimetric inequalities of a special kind. We also prove a comparison theorem for Hardy constants with different numerical parameters and we study the relationships between the linear hyperbolic isoperimetric inequality in a domain and Euclidean maximum modulus of this domain. In the proofs, an essential role is played by characteristics of domains with uniformly perfect boundary. In addition, we generalize certain results from the papers J.L. Fernández, J.M. Rodríguez, "The exponent of convergence of Riemann surfaces, bass Riemann surfaces", Ann. Acad. Sci. Fenn. Series A. I. Mathematica. 15, 165-183 (1990); V. Alvarez, D. Pestana, J.M. Rodríguez, "Isoperimetric inequalities in Riemann surfaces of infinite type", Revista Matemática Iberoamericana, 15:2, 353-425 (1999).
\end{abstract}

Keywords: Poincaré metric, hyperbolic isoperimetric inequality, uniformly perfect set, Hardy type inequality.

Mathematics Subject Classification: 30F45, 30A10

\section{INTRODUCTION}

Let $\Omega$ be a hyperbolic domain, that is, it contains at least three boundary points on the extended complex plane $\overline{\mathbb{C}}$. By $C_{0}^{1}(\Omega)$ we denote the family of continuously differentiable functions $u: \Omega \rightarrow \mathbb{R}$ compactly supported in $\Omega$. If $\infty \in \Omega$, then the smoothness of $u(z)$ at the infinity $z=\infty$ is understood as the smoothness of $u\left(\frac{1}{z}\right)$ at the point $z=0$.

At each point $z=x+i y \in \Omega$ we define a hyperbolic radius by the formula

$$
R(z, \Omega)=\frac{1}{\lambda_{\Omega}(z)},
$$

where $\lambda_{\Omega}$ is the coefficient of the Poincaré metric of the domain $\Omega$ with the Gaussian curvature $k=-4$, see, for instance, [1], [2].

According Ch. Pommerenke [3], we say that a hyperbolic domain $\Omega \subset \overline{\mathbb{C}}$ has a uniformly perfect boundary if $M(\Omega)<\infty$, where $M(\Omega)$ is the supremum of the moduli of two-connected domain lying in the domain $\Omega$ and separating its boundary. We recall that the modulus of a

F.G. Avkhadiev, R.G. Nasibullin, I.K. Shafigullin, Conformal invariants of hyperbolic PlaNAR DOMAINS.

(c)Avkhadiev F.G., Nasibullin R.G., Shafigullin I.K. 2019 .

The reported study was funded by RFBR according to the research project no. 18-11-00115).

Submitted February 20, 2019. 
two-connected domain $\Omega^{\prime}$ is defined as follows. We take an annulus $A$ defined by the inequality $r(A)<|z|<R(A)$ and conformally equivalent to the domain $\Omega^{\prime}$. The number

$$
M\left(\Omega^{\prime}\right)=\frac{1}{2 \pi} \log \frac{R(A)}{r(A)}
$$

is called the modulus of the two-connected domain $\Omega^{\prime}$. We say that a two-connected domain $\Omega^{\prime}$ separates the boundary of the domain $\Omega$ if $\Omega^{\prime} \subset \Omega$ and each component of the set $\mathbb{C} \backslash \Omega^{\prime}$ possesses points in $\partial \Omega$.

Apart of the maximal modulus $M(\Omega)$, we shall need the following scalar characteristics of a hyperbolic domain $\Omega$ :

$$
h(\Omega)=\sup _{G} \iint_{G} \frac{1}{R^{2}(z, \Omega)} d x d y\left(\int_{\partial G} \frac{1}{R(z, \Omega)}|d z|\right)^{-1},
$$

where the upper bound is taken over all domains $G$ enveloped by piecewise-smooth curves such that $\bar{G} \subset \Omega$. We also mention that a hyperbolic area $\iint_{G} R^{-2}(z, \Omega) d x d y$ and a hyperbolic length $\int_{\partial G} R^{-1}(z, \Omega)|d z|$ are dimensionless quantities. It is obvious that the condition $h(\Omega)<\infty$ means that a linear hyperbolic isoperimetric inequality holds in the domain $\Omega \subset \overline{\mathbb{C}}$.

The present work is devoted to studying new conformally invariant quantities (functionals) defined as sharp constants in variational inequalities of special form for the functions $u \in C_{0}^{1}(\Omega)$ in planar hyperbolic domains.

The main conformally invariant functional $c_{p, q}(\Omega)$ of a domain we consider is defined as the maximal among possible constants in the following Hardy type variational inequality

$$
\left(\iint_{\Omega} \frac{|\nabla u|^{p} d x d y}{R^{2-p}(z, \Omega)}\right)^{\frac{1}{p}} \geqslant c_{p, q}(\Omega)\left(\iint_{\Omega} \frac{|u|^{q} d x d y}{R^{2}(z, \Omega)}\right)^{\frac{1}{q}} \quad \text { for all } \quad u \in C_{0}^{1}(\Omega),
$$

where $1<p \leqslant q<\infty, z=x+i y, \nabla u$ is the gradient of the function $u$. Thus, the considered functional $c_{p, q}(\Omega)$ is defined by the formula

$$
c_{p, q}(\Omega)=\inf _{u \in C_{0}^{1}(\Omega), u \neq 0}\left(\iint_{\Omega} \frac{|\nabla u|^{p} d x d y}{R^{2-p}(z, \Omega)}\right)^{\frac{1}{p}}\left(\iint_{\Omega} \frac{|u|^{q} d x d y}{R^{2}(z, \Omega)}\right)^{-\frac{1}{q}} .
$$

The conformal invariance for the functional defined by formula (2) can be easily confirmed. Indeed, let $F: \Omega \rightarrow \Omega_{\zeta}$ be a univalent conformal mapping of a domain $\Omega$ on some other domain $\Omega_{\zeta} \subset \overline{\mathbb{C}}$. We denote

$$
\zeta=F(z)=\xi+i \eta \in \Omega_{\zeta}, U:=u \circ F^{-1}
$$

where $z=x+i y \in \Omega$ and $u \in C_{0}^{1}(\Omega)$. Then $U:=u \circ F^{-1} \in C_{0}^{1}\left(\Omega_{\zeta}\right)$ and the formulae hold:

$$
\begin{array}{ll}
\lambda_{\Omega}(z)|d z| \equiv \lambda_{\Omega_{\zeta}}(z)|d \zeta|, & \lambda_{\Omega}^{2}(z) d x d y=\lambda_{\Omega_{\zeta}}^{2}(z) d \xi d \eta \\
\left|F^{\prime}(z)\right|^{2} d x d y=d \xi d \eta, & \nabla U=2 \frac{\partial U \zeta}{\partial \bar{\zeta}}=2 \frac{\partial u(z)}{\partial \bar{z}} \overline{F^{\prime}(z)}=(\nabla u) \overline{F^{\prime}(z)} .
\end{array}
$$

Introducing $c_{p, q}\left(\Omega_{\zeta}\right)$ by formula (2) the domain $\Omega$ replaced by the domain $\Omega_{\zeta}$ and the function $u$ replaced by the function $U$, we obtain:

$$
c_{p, q}(\Omega)=c_{p, q}\left(\Omega_{\zeta}\right) .
$$

The basic results we use are well-known results by D. Sullivan [4], J.L. Fernández [5], J.L. Fernández and J.M. Rodríguez [6] on the spectral theory of the Laplace-Beltrami operator on Riemannian manifolds of a constant negative curvature. The cited papers were devoted to studying a particular case of inequality (1) corresponding to the case $p=q=2$, namely, the inequality

$$
\iint_{\Omega}|\nabla u|^{2} d x d y \geqslant c_{2}(\Omega) \iint_{\Omega} \frac{|u|^{2}}{R^{2}(z, \Omega)} d x d y, \quad \forall u \in C_{0}^{1}(\Omega),
$$


where

$$
c_{2}(\Omega)=\inf _{u \in C_{0}^{1}(\Omega), u \neq 0} \iint_{\Omega}|\nabla u|^{2} d x d y\left(\iint_{\Omega} \frac{|u|^{2}}{R^{2}(z, \Omega)} d x d y\right)^{-1} .
$$

It is obvious that $c_{2}(\Omega)=c_{2,2}^{2}(\Omega)$. It is known, [4], [5], that $c_{2}(\Omega)=1$ for each simply-connected or two-connected hyperbolic domain and also $c_{2}(\Omega) \in[0,1]$ for each hyperbolic domain. There exist domains, for which $c_{2}(\Omega)=0$, that is, as $p=q=2$, there exist domains, for which inequality (1) is trivial. These statements are corollaries of well-known facts in the hyperbolic geometry and the Elstrodt-Patterson-Sullivan formula [4]:

$$
c_{2}(\Omega)=\left\{1 \text { as } 0 \leqslant \beta \leqslant \frac{1}{2} ; 4 \beta(1-\beta) \text { as } \frac{1}{2} \leqslant \beta \leqslant 1\right\},
$$

where $\beta=\beta(\Omega)$ is the critical convergence exponent of the Poincaré-Dirichlet series for the group of fundamental transformations of $\Omega$.

In [5], Fernández proved that condition $M(\Omega)<\infty$ implies the positivity of the quantity $c_{2}(\Omega)$. The key result of paper [6] by Fernández and Rodgriquez are the estimates

$$
\frac{1}{(2 h(\Omega))^{2}} \leqslant c_{2}(\Omega) \leqslant \frac{3}{h(\Omega)}
$$

F.G. Avkhadiev [7]-[9] studied the following generalization of (3):

$$
\iint_{\Omega} \frac{|\nabla u|^{p}}{R^{2-p}(z, \Omega)} d x d y \geqslant c_{p}(\Omega) \iint_{\Omega} \frac{|u|^{p}}{R^{2}(z, \Omega)} d x d y, \quad \forall u \in C_{0}^{1}(\Omega),
$$

where $p \in[1, \infty)$ is a fixed number and

$$
c_{p}(\Omega)=\inf _{u \in C_{0}^{1}(\Omega), u \neq 0} \iint_{\Omega} \frac{|\nabla u|^{p}}{R^{2-p}(z, \Omega)} d x d y \iint_{\Omega} \frac{|u|^{p}}{R^{2}(z, \Omega)} d x d y .
$$

It was proved in [7]-[9] that the condition $M(\Omega)<\infty$ implies the positivity of the quantity $c_{p}(\Omega)$ for each $p \in[1, \infty)$ and the identity $c_{p}(\Omega)=2^{p} / p^{p}$ was established for each simply-connected or two-connected hyperbolic domain for each $p \in[1, \infty)$. Moreover, in [9], there were proved the estimates for the constant $c_{p}(\Omega)$ depending on the maximal Euclidean modulus $M_{0}(\Omega)$ and the exponent $p \in[1, \infty)$. We note that

$$
M_{0}(\Omega) \leqslant M(\Omega) \leqslant M_{0}(\Omega)+1 / 2
$$

for domains $\Omega \subset \mathbb{C}$, see [2] for more details, and

$$
M_{0}(\Omega) \leqslant M(\Omega) \leqslant 2 M_{0}(\Omega)+1
$$

for domains $\Omega \subset \overline{\mathbb{C}}, \infty \in \Omega$, see [9], where $M(\Omega)$ is the maximal modulus introduced above.

In the present paper we obtain some new estimates for the constant $c_{p}(\Omega)$ and we generalize them for $c_{p, q}(\Omega)$ as $1 \leqslant p \leqslant q<\infty$. In particular, we show that for $p \in[1,2)$, the inequality $c_{p}(\Omega)>0$ holds if and only if the coefficient $h(\Omega)$ of the linear isoperimetric inequality for the hyperbolic metric is positive. We also prove that if the quantity

$$
h_{p, q}(\Omega)=\sup _{G}\left(\iint_{G} \frac{1}{R^{2}(z, \Omega)} d x d y\right)^{\frac{1}{q}-\frac{1}{p}+1}\left(\int_{\partial G} \frac{1}{R(z, \Omega)}|d z|\right)^{-1}
$$

is finite as $\frac{1}{p}-1 / 2 \leqslant \frac{1}{q} \leqslant \frac{1}{p} \leqslant 1$, this implies the positivity of the constant $c_{p, q}(\Omega)$. We note that some results concerning $c_{p}(\Omega)$ and provided here with the full proofs, were announced before in a short letter [10].

In the case $\infty \notin \Omega$, several criterions for the complete perfectness of the boundary are known in terms of the hyperbolic radius $R(z, \Omega)$, its gradient $\nabla R(z, \Omega)$ and the distance dist $(z, \partial \Omega)$ 
to the boundary of the domain $\Omega$, see [3], [9]. We shall estimate $h(\Omega)$ in terms of these characteristics. For instance, we shall prove that

$$
\sqrt{3} \sup _{z \in \Omega}|\nabla R(z, \Omega)|>2 \sqrt{h(\Omega)} .
$$

We observe the following fact. The weighted functions considered in the present paper involve the hyperbolic radius $R(z, \Omega)$. There are many close works devoted to Hardy type inequalities, in which the weight function involves the distance dist $(z, \partial \Omega)$ to the boundary of the domain, see [11]-[20]. It should be also stressed that the one-dimensional Hardy inequality are not related with the geometry. They are regarded as some tool from the theory of functions employed in the proofs of embedding theorems for functional spaces, cf. the monographs by S.L. Sobolev [21] and V.G. Maz'ya [22]. In contrast to the one-dimensional case, the Hardy type inequality on planar domains are a part of the geometric analysis since they are essentially related with various geometric characteristics.

For the reader's convenience, in the next section we provide known results, which will be employed essentially in our proofs.

\section{AUXILIARY STATEMENTS AND DEFINITIONS}

In the proofs, the approach by V.M. Miklyukov and M.K. Vuorinen [23] plays an essential role; this approach is related with an isoperimetric profile of a domain.

Let $\Omega$ be a hyperbolic domain on the extended plane and let $\alpha, \beta: \Omega \rightarrow(0, \infty)$ be some continuous functions. We consider fixed parameters $p$ and $q$ satisfying the condition $1<p \leqslant$ $q<\infty$. On the set of the domains $G \subset \Omega$ such that the boundary $\partial G$ consists of piece-wise smooth curves and $\bar{G} \subset \Omega$, we define a weighted area

$$
V(G)=\iint_{G} \alpha(z)^{q} d x d y
$$

and a weighted length

$$
A(G)=\int_{\partial G} \beta(z) \alpha(z)^{(p-1) q / p}|d z| .
$$

An isoperimetric profile of a planar domain $\Omega$ is the best possible (maximal) function

$$
\theta:[0, V(\Omega)) \rightarrow[0, \infty), \quad \theta(0)=0,
$$

obeying the condition

$$
\theta(V(G)) \leqslant A(G)
$$

for each admissible domain $G$, i.e., for each domain with a piece-wise smooth boundary such that $\bar{G} \subset \Omega$.

Let us provide the formulation of the main statement in paper [23] by V.M. Miklyukov and M.K. Vuorinen [23] in a generality we need. It should be noted that in paper [23], more special conditions for the functions $\alpha$ and $\beta$ are assumed which are not employed in the proof but simplify the description of various applications.

Theorem A. Let $1<p \leqslant q<\infty$ and let $\Omega$ be a hyperbolic domain on the extended plane $\overline{\mathbb{C}}$. If for the domain $\Omega$ there exists an isoperimetric profile satisfying the relation

$$
B:=\sup _{r \in(0, V(\Omega))} r^{\frac{1}{q}}\left(\int_{r}^{V(\Omega)} \theta(t)^{-\frac{p}{p-1}} d t\right)^{\frac{p-1}{p}}<\infty,
$$


then for each function $u \in C_{0}^{1}(\Omega)$, the following inequality

$$
\left(\iint_{\Omega}|\alpha(z) u(z)|^{q} d x d y\right)^{\frac{1}{q}} \leqslant \lambda\left(\iint_{\Omega}(\beta(z)|\nabla u(z)|)^{p} d x d y\right)^{\frac{1}{p}}
$$

holds true, where $z=x+i y$ and $\lambda$ is a positive constant satisfying the estimates:

$$
B \leqslant \lambda \leqslant B q^{\frac{1}{q}}\left(\frac{q}{(q-1)}\right)^{\frac{p-1}{p}} .
$$

We shall also need the following three theorems by Fernández and Rodríguez.

Theorem B. (J.L. Fernández, J.M. Rodríguez [6]) Let $\Omega$ be a hyperbolic domain. The inequality $h(\Omega)<\infty$ is true if and only if $c_{2}(\Omega)>0$, moreover,

$$
\frac{1}{(2 h(\Omega))^{2}} \leqslant c_{2}(\Omega) \leqslant \frac{3}{h(\Omega)} \text {. }
$$

The next theorem is devoted to the domain with uniformly perfect boundary.

Theorem C. (J.L. Fernández, J.M. Rodríguez [6]) Let $\Omega \subset \overline{\mathbb{C}}$ be a hyperbolic domain such that $M(\Omega)<\infty$. Let $A \subset \Omega$ be a domain consisting of finitely many or countably many points such that

$$
\inf _{z \in A, w \in A \backslash\{z\}} d_{\Omega}(z, w)>0,
$$

where $d_{\Omega}(z, w)$ is the hyperbolic distance between the points $z, w \in \Omega$. Then

$$
c_{2}(\Omega \backslash A)>0 \text {. }
$$

As it was pointed out in paper [24], as the set $A$ in this theorem, we can choose an arbitrary set $A \subset \Omega$ consisting of finitely many points.

Theorem D. (J.L. Fernández, J.M. Rodríguez [6]) Let $\Omega$ be a planar domain, $\infty \in \Omega$ such that $c_{2}(\Omega)>0$ and $I$ is a set of isolated points in $\partial \Omega$. Then the points $I$ are uniformly separated.

In [24], Alvarez, Pestana and Rodríguez obtained statements generalizing corresponding results by Fernández and Rodríguez from [6]. We note that they extended the results from [6] to the case of hyperbolic Riemannian surfaces and some of these results are new even for planar domains. Let us provide one of these statements.

Theorem E. (V. Alvarez, D. Pestana, J.M. Rodríguez, [24]). Let $\Omega$ be a hyperbolic domain, $I$ be a closed countable subset of $\Omega$ and $R=\Omega \backslash I$. The inequality $h(R)<\infty$ holds if and only if $h(\Omega)<\infty$ and for some fixed number $r_{0}>0$, at each point $t \in I$ there exist simply-connected and mutually disjoint hyperbolic balls $B_{\Omega}\left(t, r_{0}\right)$ of radius $r_{0}$ centered at $t$. The estimate holds:

$$
h(R) \leqslant \frac{h(\Omega)}{\tanh ^{2}\left(\frac{r_{0}}{4}\right)}+\frac{2 \pi}{r_{0} \log \frac{\tanh r_{0}}{\tanh \left(\frac{r_{0}}{4}\right)} .}
$$

Before we formulate the next results by F.G. Avkhadiev from [9], we introduce some notations. The maximal Euclidean modulus is introduced by the identity

$$
M_{0}(\Omega):=\sup \frac{1}{2 \pi} \log \frac{R(A)}{r(A)},
$$

where the supremum is taken over all annuli $A$ such that $A$ separates the boundary $\Omega$,

$$
A=\left\{z \in \mathbb{C}: r(A)<\left|z-z_{0}\right|<R(A)\right\} \subset \Omega \quad \text { and } \quad z_{0} \in \partial \Omega .
$$

We let $M_{0}(\Omega)=0$, if the set of all such annuli is an empty set. 
The following statement holds.

Theorem F. (F.G. Avkhadiev [9]) Let $1 \leqslant p<\infty$. If $\Omega \subset \overline{\mathbb{C}}$ is a domain with a uniformly perfect boundary containing at least three components, then for each real-valued function $u \in$ $C_{0}^{1}(\Omega)$ the inequality holds:

$$
\iint_{\Omega} \frac{|\nabla u|^{p} d x d y}{R^{2-p}(x+i y, \Omega)} \geqslant \frac{1}{p^{p} \mu^{p}(\Omega)} \iint_{\Omega} \frac{|u|^{p} d x d y}{R^{2}(x+i y, \Omega)},
$$

where

Here $\Gamma$ is the Euler gamma function.

$$
\mu(\Omega)= \begin{cases}\pi M_{0}(\Omega)+\frac{\Gamma^{4}\left(\frac{1}{4}\right)}{4 \pi^{2}}, & \text { if } \infty \notin \Omega, \\ 2 \pi M_{0}(\Omega)+\pi+\frac{\Gamma^{4}\left(\frac{1}{4}\right)}{4 \pi^{2}}, & \text { if } \infty \in \Omega .\end{cases}
$$

\section{MAIN RESULTS}

First we prove the comparison theorem for the constants $c_{r}(\Omega)$ for different $r$. Close results on Hardy inequalities of another type were presented in our papers [8] and [17].

Theorem 1. Let $1 \leqslant p \leqslant r<\infty$ and let $\Omega \subset \overline{\mathbb{C}}$ be a hyperbolic domain. Then

$$
c_{r}(\Omega) \geqslant \frac{p^{r}\left[c_{p}(\Omega)\right]^{\frac{r}{p}}}{r^{r}} .
$$

Proof. As $p=r$, relation (7) becomes an identity. This is why we consider only the case $p<r$.

Let $u \in C_{0}^{1}(\Omega), u \neq \equiv 0$ and $1 \leqslant p<r<\infty$. We define a new function $\varphi: \Omega \rightarrow \mathbb{R}$ by the identity $\varphi(z) \equiv|u(z)|^{r / p}, z=x+i y \in \Omega$. It is obvious that $\varphi \in C_{0}(\Omega)$. We have

$$
\nabla \varphi(z)=(r / p)|u(z)|^{r / p-1}(\operatorname{sign} u(z)) \nabla u(x) .
$$

Since $\frac{r}{p}-1>0$ and $u \in C_{0}^{1}(\Omega)$, the function $\varphi$ is continuously differentiable at the points $z \in \Omega$, where $u(z) \neq 0$. If $u\left(z_{0}\right)=0$ at some point $z_{0} \in \Omega$, then it is clear that $\nabla \varphi\left(z_{0}\right)=0$ and $\lim _{z \rightarrow z_{0}} \nabla \varphi(z)=0$ thanks to the relations $\frac{r}{p}-1>0$ and since $u \in C_{0}^{1}(\Omega)$. Hence, we get: $\varphi=|u|^{\frac{r}{p}} \in C_{0}^{1}(\Omega)$.

Applying inequality (5) to the function $\varphi$, we obtain:

$$
\frac{r^{p}}{p^{p}} \iint_{\Omega} \frac{|u|^{r-p}|\nabla u|^{p}}{R^{2-p}(z, \Omega)} d x d y \geqslant c_{p}(\Omega) \iint_{\Omega} \frac{|u|^{r}}{R^{2}(z, \Omega)} d x d y, \quad u \in C_{0}^{1}(\Omega) .
$$

Let us estimate from above the integral in the left hand side of this inequality. Letting

$$
p_{1}=\frac{r}{r-p}, \quad p_{2}=\frac{r}{p}, \quad f_{1}=\frac{|u|^{r-p}}{R^{2-2 \frac{p}{r}}}, \quad f_{2}=\frac{|\nabla u|^{p}}{R^{2 \frac{p}{r}-p}},
$$

and applying the Hölder inequality

$$
\iint_{\Omega} f_{1} f_{2} d x d y \leqslant\left(\iint_{\Omega} f_{1}^{p_{1}} d x d y\right)^{\frac{1}{p_{1}}}\left(\iint_{\Omega} f_{2}^{p_{2}} d x d y\right)^{\text {frac1 } p_{2}} .
$$

As a result, we arrive at the inequality

$$
\frac{r^{p}}{p^{p}}\left(\iint_{\Omega} \frac{|u|^{r} d x d y}{R^{2}(z, \Omega)}\right)^{1-\frac{p}{r}}\left(\iint_{\Omega} \frac{|\nabla u|^{r} d x d y}{R^{2-r}(z, \Omega)}\right)^{\frac{p}{r}} \geqslant c_{p}(\Omega) \iint_{\Omega} \frac{|u|^{r} d x d y}{R^{2}(z, \Omega)} .
$$

Since $u \in C_{0}^{1}(\Omega)$ and $u \not \equiv 0$, this inequality is equivalent to the following one:

$$
\iint_{\Omega} \frac{|\nabla u|^{r} d x d y}{R^{2-r}(z, \Omega)} \geqslant \frac{p^{r}\left[c_{p}(\Omega)\right]^{\frac{r}{p}}}{r^{r}} \iint_{\Omega} \frac{|u|^{r} d x d y}{R^{2}(z, \Omega)}, \quad \text { for all } \quad u \in C_{0}^{1}(\Omega), \quad u \neq \equiv .
$$


This implies inequality (7) since in view of definition (6), as $r=p$, the constant $c_{r}(\Omega)$ is the maximal possible in the inequality

$$
\iint_{\Omega} \frac{|\nabla u|^{r} d x d y}{R^{2-r}(z, \Omega)} \geqslant c_{r}(\Omega) \iint_{\Omega} \frac{|u|^{r} d x d y}{R^{2}(z, \Omega)}, \quad \text { for all } \quad u \in C_{0}^{1}(\Omega)
$$

The proof is complete.

The next two statements generalize Theorem B by Fernández and Rodríguez. We obtain estimates for conformally invariant quantities $c_{p}(\Omega)$ and $c_{p, q}(\Omega)$ under some restrictions for the parameters $p$ and $q$. We recall that the quantities $c_{p}(\Omega)$ and $c_{p, q}(\Omega)$ are comparable with the constant $c_{2}(\Omega)$ under the condition $p=q=2$. In particular, as $p=2$, the next theorem coincides with Theorem B by Fernández and Rodríguez.

Theorem 2. Let $\Omega \subset \overline{\mathbb{C}}$ be a hyperbolic domain with the coefficient in the linear isoperimetric inequality defined by the identity:

$$
h(\Omega)=\sup _{G} \iint_{G} R^{-2}(z, \Omega) d x d y\left(\int_{\partial G} R^{-1}(z, \Omega)|d z|\right)^{-1},
$$

where the supremum is taken over all domains $G$ compactly embedded in the domain $\Omega$ and enveloped by piece-wise smooth curves.

The following statements hold true.

1) If $h(\Omega)<\infty$, then the constant $c_{p}(\Omega)$ is a positive number for each $p \in[1, \infty)$ and the estimate holds: $c_{p}(\Omega) \geqslant \frac{1}{(p h(\Omega))^{p}}$.

2) For each $p \in[1,2]$, the constant $c_{p}(\Omega)$ is positive if and only $h(\Omega)<\infty$. The estimates holds:

$$
\frac{1}{h^{p}(\Omega)} \leqslant p^{p} c_{p}(\Omega) \leqslant \frac{12^{\frac{p}{2}}}{h^{\frac{p}{2}}(\Omega)} .
$$

Proof. We begin with proving the first statement of the theorem. Assume that $p \in(1, \infty)$ and $h(\Omega)<\infty$. By the definition of the conformally invariant constant $h(\Omega)$, for each domain $G$ compactly embedded into $\Omega$ and enveloped by piece-wise smooth curves we have

$$
V(G):=\iint_{G} \frac{d x d y}{R^{2}(z, \Omega)} \leqslant h(\Omega) \int_{\partial G} \frac{d s}{R(z, \Omega)} .
$$

Then we apply Theorem A by V.M. Miklyukov and M.K. Vuorinen letting $q=p \in(1, \infty)$,

$$
\alpha(z)=R^{-\frac{2}{p}}(z, \Omega), \quad \beta(z)=R^{-\frac{2}{p}+1}(z, \Omega) .
$$

It follows from the definition of the isoperimetric profile of the domain $\Omega$ that the profile satisfies the inequality

$$
\theta(t) \geqslant \frac{t}{h(\Omega)}
$$

for each $t \in\left(0, I_{p}\right)$, where $I_{p}=\sup _{G} V(G)$. This is why

$$
B:=\sup _{r \in\left(0, I_{p}\right)} r^{\frac{1}{p}}\left(\int_{r}^{I_{p}} \theta(t)^{-\frac{p}{p-1}} d t\right)^{\frac{p-1}{p}} \leqslant h(\Omega) \sup _{r \in(0, \infty)} r^{\frac{1}{p}}\left(\int_{r}^{\infty} t^{-\frac{p}{p-1}} d t\right)^{\frac{p-1}{p}}=h(\Omega)(p-1)^{1-\frac{1}{p}} .
$$

The above identity has been obtained by means of straightforward calculations.

By Theorem A with $q=p \in(1, \infty)$, we have the inequality:

$$
\iint_{\Omega} \frac{|\nabla u|^{p}}{R^{2-p}(z, \Omega)} d x d y \geqslant \lambda^{-p} \iint_{\Omega} \frac{|u|^{p}}{R^{2}(z, \Omega)} d x d y, \quad \text { for all } \quad u \in C_{0}^{1}(\Omega),
$$


where the constant $\lambda$ satisfies the inequality

$$
\lambda \leqslant B p^{\frac{1}{p}}\left(\frac{p}{p-1}\right)^{\frac{p-1}{p}} \leqslant p h(\Omega) .
$$

Now we observe that the constant $c_{p}(\Omega)$ is defined as the maximal constant in inequality (9). Therefore,

$$
c_{p}(\Omega) \geqslant \lambda^{-p}
$$

This estimate and (10) lead us to the inequalities

$$
c_{p}(\Omega) \geqslant \frac{1}{(p h(\Omega))^{p}}>0 .
$$

This proves the first statement of the theorem for each $p \in(1, \infty)$.

It remains to consider the case $p=1$. We choose a function $u \in C_{0}^{1}(\Omega)$. For each $p \in(1, \infty)$, this function satisfies inequality (9) with a constant $\lambda$ obeying estimate (10). Since the integrals in inequalities (9) depend continuously on the parameter $p \in(1, \infty)$ for a fixed function $u \in$ $C_{0}^{1}(\Omega)$, we can pass to the limit as $p \rightarrow 1$. It is clear that by passing to the limit as $p \rightarrow 1$ in (9) and (10), we arrive at the estimate

$$
c_{1}(\Omega) \geqslant \frac{1}{h(\Omega)}>0
$$

in view of the definition of $c_{1}(\Omega)$ as a maximal constant in the corresponding inequality.

Let us prove the second statement. Assume that $p \in[1,2]$. If $h(\Omega)<\infty$, the positivity of $c_{p}(\Omega)$ and the lower bound for this quantity follow the first statement of the theorem.

Assume that $c_{p}(\Omega)>0$ for a fixed $p \in[1,2]$. As $p=2$, the inequality $h(\Omega)<\infty$ and the upper bound

$$
c_{2}(\Omega) \leqslant \frac{3}{h(\Omega)}
$$

were proved by Fernández and Rodríguez, see Theorem $B$. Suppose that $p \in[1,2)$ and $c_{p}(\Omega)>$ 0 . Applying estimate (7) of Theorem 1 as $r=2$, we have:

$$
c_{2}(\Omega) \geqslant \frac{p^{2}\left[c_{p}(\Omega)\right]^{\frac{2}{p}}}{4}>0 .
$$

Applying this estimate and Theorem $\mathrm{B}$, we get that $h(\Omega)<\infty$ and

$$
c_{p}(\Omega) \leqslant\left(\frac{4 c_{2}(\Omega)}{p^{2}}\right)^{\frac{p}{2}} \leqslant\left(\frac{12}{h(\Omega) p^{2}}\right)^{\frac{p}{2}} .
$$

The proof is complete.

We provide several corollaries of Theorem 2 and the aforementioned theorems by Fernández, Rodríguez and Avkhadiev.

Corollary 1. Let $\Omega \subset \overline{\mathbb{C}}$ be a domain with a uniformly perfect boundary. Then the following estimate holds true:

$$
\sqrt{h(\Omega)}<2 \sqrt{3} \mu(\Omega)
$$

where

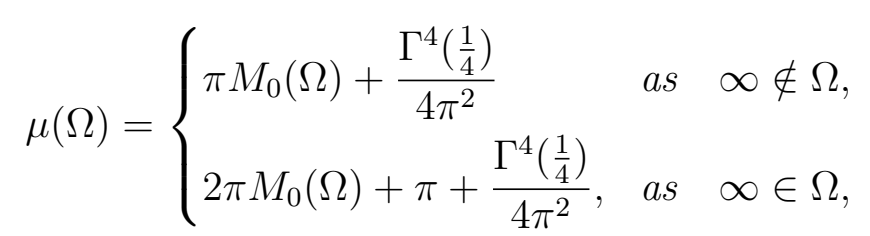

where $\Gamma$ is the Euler gamma function. 
Proof. By Avkhadiev theorem F, for a hyperbolic domain with a uniformly perfect boundary we obtain

$$
c_{p}(\Omega) \geqslant \frac{1}{p^{p} \mu(\Omega)} .
$$

As $p \in[1,2)$, by Theorem 2 we have

$$
\left(\frac{12}{p^{2} h(\Omega)}\right)^{\frac{p}{2}}>c_{p}(\Omega) \geqslant \frac{1}{p^{p} \mu^{p}(\Omega)} .
$$

Therefore,

$$
\sqrt{h(\Omega)}<\sqrt{12} \mu(\Omega)
$$

and this completes the proof.

In the next statement, the symbol $d_{\Omega}(z, w)$ denotes the hyperbolic distance between the points $z, w \in \Omega$.

Corollary 2. Let $\Omega \subset \overline{\mathbb{C}}$ be a hyperbolic domain such that $M(\Omega)<\infty$, that is, the boundary of the domain $\Omega$ is a uniformly perfect set. Let $A \subset \Omega$ be a set consisting of finitely many or countably many points and $R=\Omega \backslash A$. If $A$ is a countable set, we assume that

$$
\inf _{z \in A, w \in A \backslash\{z\}} d_{\Omega}(z, w)>0 .
$$

Then $c_{p}(R)>0$ as $1 \leqslant p<\infty$.

Proof. It follows from Fernández-Rodríguez theorem [6] that $c_{2}(R)>0$. Hence, by Theorem $\mathrm{B}$, the isoperimetric constant satisfies $h(R)<\infty$. Applying now Theorem 2 , we get

$$
c_{p}(R) \geqslant \frac{1}{h(R)^{p} p^{p}}>0
$$

for each $1 \leqslant p<\infty$ and this completes the proof.

Corollary 3. Let $p \in[1,2)$ and $\Omega \subset \overline{\mathbb{C}}$ be a hyperbolic domain $\infty \in \Omega$. By I we denote the set of isolated points in the boundary $\partial \Omega$. Assume that $c_{p}(\Omega)>0$. Then the points of in the set $I$ are uniformly separated in the hyperbolic metric of the domain $G=\Omega \cup I$.

Proof. By the assumption, $c_{p}(\Omega)>0$ as $p \in[1,2)$. Applying inequality (7), we obtain that $c_{2}(\Omega)>0$. Now the statement follows Theorem D by Fernández and Rodríguez.

The next statement is a corollary of Theorem 2 and the above formulated theorem by Alvarez, Pestana and Rodríguez in [24].

Corollary 4. Let $p \in[1,2)$ and $\Omega \subset \overline{\mathbb{C}}$ be a hyperbolic domain, $\infty \in \Omega, I$ be a closed countable subset of $\Omega$ and $R=\Omega \backslash I$. Then the following statements hold:

1. If $c_{p}(R)>0$, then $h(\Omega)<\infty, c_{p}(\Omega)>0$, and for some number $r_{0}>0$, at each point $t \in I$ there exist simply-connected and mutually disjoint hyperbolic circles $B_{\Omega}\left(t, r_{0}\right)$ of radius $r_{0}$ centered at $t$;

2. If $c_{p}(\Omega)>0$ and for some fixed number $r_{0}>0$ at each point $t \in I$ there exist simplyconnected and mutually disjoint hyperbolic circles $B_{\Omega}\left(t, r_{0}\right)$ of radius $r_{0}$ centered at $t$, then $h(R)<\infty$ and $c_{p}(R)>0$. Moreover, the estimate holds:

$$
c_{p}(R) \geqslant\left[\frac{\sqrt[p]{c_{p}(\Omega)}}{\tanh ^{2}\left(\frac{r_{0}}{4}\right)}+\frac{2 p \pi}{r_{0} \log \frac{\tanh r_{0}}{\tanh \left(\frac{r_{0}}{4}\right)}}\right]^{-p} .
$$


Proof. Let $c_{p}(R)>0$. Applying Theorem 2 with $p \in[1,2)$, we get $h(R)<\infty$. Then we employ Theorem E by Alvarez-Pestana-Rodríguez to obtain that $h(\Omega)<\infty$ and at each point $t \in I$ there exist simply-connected and mutually disjoint hyperbolic circles $B_{\Omega}\left(t, r_{0}\right)$ of radius $r_{0}$ centered at $t$. It remains to apply Theorem 2 once again to obtain inequality $c_{p}(\Omega)>0$.

Let $c_{p}(\Omega)>0$. Then by Theorem 2 we have the inequality $h(\Omega)<\infty$ and the estimate

$$
p h(\Omega) \geqslant\left[c_{p}(\Omega)\right]^{\frac{1}{p}} .
$$

Since at each point $t \in I$ there exist simply-connected and mutually disjoint hyperbolic circles $B_{\Omega}\left(t, r_{0}\right)$ of radius $r_{0}$ centered at $t$, by Theorem $E$ we obtain that $h(R)<\infty$ and moreover, the relations hold:

$$
\begin{aligned}
& h(R) \leqslant \frac{h(\Omega)}{\tanh ^{2}\left(\frac{r_{0}}{4}\right)}+\frac{2 \pi}{r_{0} \log \frac{\tanh r_{0}}{\tanh \left(\frac{r_{0}}{4}\right)},} \\
& c_{p}(R) \geqslant \frac{1}{h(R)^{p} p^{p}}>0 .
\end{aligned}
$$

Combining inequalities (11), (12) and (13), we obtain the needed statement.

Remark. According Theorem 2, if $p \in[1,2]$ and $c_{p}(\Omega)>0$, then the coefficient $h(\Omega)$ is finite. The following question remains open: whether the positivity of the constant $c_{p}(\Omega)$ for some $p \in(2, \infty)$ ensures the finiteness of the coefficient $h(\Omega)$ ? This problem can be reformulated as follows: whether there exists a hyperbolic domain $\Omega \subset \overline{\mathbb{C}}$ such that $c_{2}(\Omega)=0$ but the constant $c_{p}(\Omega)$ is positive for some $p \in(2, \infty)$.

The next theorem generalize the first statement of Theorem 2 .

Theorem 3. Assume that $\Omega \subset \overline{\mathbb{C}}$ is a hyperbolic domain, numbers $p \in[1, \infty)$ and $q \in[1, \infty)$ are fixed and satisfy the inequalities $\frac{1}{p}-\frac{1}{2} \leqslant \frac{1}{q} \leqslant \frac{1}{p}$, while the quantity $h_{p, q}(\Omega)$ is defined by the identity:

$$
h_{p, q}(\Omega)=\sup _{G}\left(\iint_{G} R^{-2}(z, \Omega) d x d y\right)^{\frac{1}{q}-\frac{1}{p}+1}\left(\int_{\partial G} R^{-1}(z, \Omega)|d z|\right)^{-1},
$$

where the supremum is taken over all domains $G$ compactly embedded into the domain $\Omega$ and enveloped by piece-wise smooth curves.

If $h_{p, q}(\Omega)<\infty$, then the constant $c_{p, q}(\Omega)$ is positive and the estimates hold:

$$
c_{p, q}(\Omega) \geqslant \frac{q^{\frac{1}{p}-\frac{1}{q}-1}}{h_{p, q}(\Omega)}\left(\frac{p(q-1)}{q(p-1)}\right)^{\frac{p-1}{p}} \quad \text { as } \quad p>1, \quad c_{1, q}(\Omega) \geqslant \frac{1}{q^{\frac{1}{q}} h_{1, q}(\Omega)} \quad \text { as } \quad p=1 .
$$

Proof. We apply the same method as in the proof of the first statement in the previous theorem. We define continuous functions $\alpha: \Omega \rightarrow(0, \infty)$ and $\beta: \Omega \rightarrow(0, \infty)$ by the identities $\alpha(z)=$ $R^{-\frac{2}{q}}(z, \Omega)$ and $\beta(z)=R^{-\frac{2}{p}+1}(z, \Omega)$, where $z=x+i y \in \Omega$.

Let $G$ be a domain enveloped by a piece-wise smooth curve and satisfying the condition $\bar{G} \subset \Omega$. Employing Miklyukov-Vuorinen definitions for the chosen functions $\alpha$ and $\beta$, we obtain the following formulae for the weighted area of the domain

$$
V(G)=\iint_{G} \alpha(z)^{q} d x d y=\iint_{G} \frac{d x d y}{R^{2}(z, \Omega)}
$$

and the weighted length of the boundary

$$
A(G)=\int_{\partial G} \beta(z) \alpha(z)^{(p-1) q / p}|d z|=\int_{\partial G} \frac{|d z|}{R(z, \Omega)} .
$$


By the assumptions of the theorem, for each admissible domain we have the inequality

$$
V^{\beta}(G) \leqslant h_{p, q}(\Omega) A(G),
$$

where $h_{p, q}(\Omega)<\infty$ and $\beta:=\frac{1}{q}-\frac{1}{p}+1 \in[1 / 2,1]$. On the other hand, the isoperimetric profile

$$
\theta:[0, V(\Omega)) \rightarrow[0, \infty), \quad \theta(0)=0
$$

of the domain $\Omega$ is the maximal function obeying the inequality

$$
\theta(V(G)) \leqslant A(G)
$$

on the set of all admissible domains $G$. Therefore,

$$
\theta(t) \geqslant t^{\beta} / h_{p, q}(\Omega) \text {. }
$$

Suppose that

$$
p \in(1, \infty), I_{p}=\sup _{G} V(G)
$$

and apply Miklyukov-Vuorinen theorem. Since

$$
\frac{\beta p}{p-1}=1+\frac{p}{q(p-1)}
$$

for each positive number $r$ we have

$$
\left(\int_{r}^{\infty} t^{-\frac{\beta p}{p-1}} d t\right)^{\frac{p-1}{p}}=\left(q\left(1-\frac{1}{p}\right)\right)^{\frac{p-1}{p}} \frac{1}{r^{\frac{1}{q}}} .
$$

The Miklyukov-Vuorinen characteristics $B$ admits the estimate

$$
\begin{aligned}
B & \leqslant \sup _{r \in\left(0, I_{p}\right)} r^{\frac{1}{q}}\left(\int_{r}^{I_{p}} \theta(t)^{-\frac{p}{p-1}} d t\right)^{\frac{p-1}{p}} \\
& \leqslant h_{p, q}(\Omega) \sup _{r \in(0, \infty)} r^{\frac{1}{q}}\left(\int_{r}^{\infty} t^{-\frac{\beta p}{p-1}} d t\right)^{\frac{p-1}{p}}=h_{p, q}(\Omega)\left(\frac{q(p-1)}{p}\right)^{\frac{p-1}{p}} .
\end{aligned}
$$

Therefore, by Theorem A, the following inequality holds:

$$
\left(\iint_{\Omega} \frac{|\nabla u|^{p}}{R^{2-p}(z, \Omega)}\right)^{\frac{1}{p}} d x d y \geqslant \frac{1}{\lambda}\left(\iint_{\Omega} \frac{|u|^{p}}{R^{2}(z, \Omega)} d x d y\right)^{\frac{1}{q}} \quad \text { for all } \quad u \in C_{0}^{1}(\Omega) .
$$

The constant in the latter inequality obeys the relation

$$
\lambda \leqslant B q^{\frac{1}{q}}\left(\frac{q}{q-1}\right)^{\frac{p-1}{p}} \leqslant h_{p, q}(\Omega) q^{\frac{1}{q}-\frac{1}{p}+1}\left(\frac{q(p-1)}{p(q-1)}\right)^{\frac{p-1}{p}} .
$$

Since the constant $c_{p, q}(\Omega)$ is introduced as the maximal in inequality $(14)$, then $c_{p, q}(\Omega) \geqslant \lambda^{-1}$. Due to estimate (15), we obtain the desired inequality for $c_{p, q}(\Omega)$ in the case $p>1$.

The case $p=1$ can be proved by passing to the limit as in hte proof of the first statement in the previous theorem since in inequalities (14) and (15) we can pass to the limit as $p \rightarrow 1$ for a fixed function $u \in C_{0}^{1}(\Omega)$. The proof is complete.

We provide one more corollary of Theorem 3 on the case $p=1$.

Corollary 5. Assume that $\Omega \subset \overline{\mathbb{C}}$ is a hyperbolic domain. If $1 \leqslant q \leqslant 2$ and $h_{1, q}(\Omega)<\infty$, then

$$
h_{1, q}(\Omega) q^{\frac{1}{q}} \iint_{\Omega} \frac{|\nabla u|}{R(z, \Omega)} d x d y \geqslant\left(\iint_{\Omega} \frac{|u|^{q}}{R^{2}(z, \Omega)} d x d y\right)^{\frac{1}{q}} \quad \text { for all } \quad u \in C_{0}^{1}(\Omega) .
$$


Apart of the coefficient $h(\Omega)$ and the Euclidean maximal modulus $M_{0}(\Omega)$, in what follows we shall need some other scalar characteristics of hyperbolic domains, namely, $\alpha(\Omega), \gamma(\Omega)$ and $C(\Omega)$; we shall give their definitions below.

These characteristics are mutually related. It is known (see, for instance, [2], [3], [7]-[9]) that a hyperbolic domain $\Omega \subset \mathbb{C}$ possesses a uniformly perfect boundary if and only if

$$
M_{0}(\Omega)<\infty \Longleftrightarrow \alpha(\Omega)>0 \Longleftrightarrow \gamma(\Omega)<\infty \Longleftrightarrow C(\Omega)>0,
$$

where

$$
\begin{gathered}
\alpha(\Omega):=\inf _{z \in \Omega} \frac{\operatorname{dist}(z, \partial \Omega)}{R(z, \Omega)}, \quad \gamma(\Omega):=\sup _{z \in \Omega}|\nabla R(z, \Omega)|, \\
C(\Omega):=\inf \left\{\frac{\operatorname{cap}\left(\left\{\left|z-z_{0}\right| \leqslant r\right\}\right) \cap(\mathbb{C} \backslash \Omega)}{r}: z_{0} \in \partial \Omega, 0<r<\infty\right\} .
\end{gathered}
$$

The symbol cap $E$ stands for the logarithmic capacity of a domain $E$, see, for instance, [3].

Theorem 4. Let $\Omega \subset \mathbb{C}$ be a domain with a uniformly perfect boundary. Then

$$
\sqrt{h(\Omega)} \leqslant \sqrt{3}\left(\log \frac{1}{C(\Omega)}+\frac{\Gamma^{4}\left(\frac{1}{4}\right)}{2 \pi^{2}}\right), \quad \sqrt{h(\Omega)} \leqslant \frac{\sqrt{3} \gamma(\Omega)}{2}, \quad \sqrt{h(\Omega)}<\frac{\sqrt{3}}{\alpha(\Omega)} .
$$

Here $\Gamma$ is the Euler gamma function.

Proof. In our notations, for $C(\Omega)$, the inequality holds:

$$
M_{0}(\Omega) \leqslant \frac{1}{2 \pi} \log \frac{1}{C(\Omega)} \leqslant 2 M_{0}(\Omega)+\frac{4 \log 2}{\pi},
$$

see [3], [7] for more details. Employing this relation and Corollary 1, we get:

$$
\sqrt{h(\Omega)} \leqslant 2 \sqrt{3}\left(\frac{1}{2} \log \frac{1}{C(\Omega)}+\frac{\Gamma^{4}\left(\frac{1}{4}\right)}{4 \pi^{2}}\right) .
$$

If $\Omega \subset \mathbb{C}$ is a domain with a uniformly perfect boundary, then

$$
\gamma(\Omega):=\sup _{z \in \Omega}|\nabla R(z, \Omega)|<\infty
$$

and for each real-valued function $f \in C_{0}^{1}(\Omega)$ the inequality holds [9, Cor. 4.1]:

$$
\iint_{\Omega} \frac{|\nabla f|^{p} d x d y}{R^{2-p}(x+i y, \Omega)} \geqslant \frac{4^{p}}{p^{p} \gamma^{p}(\Omega)} \iint_{\Omega} \frac{|f|^{p} d x d y}{R^{2}(x+i y, \Omega)} .
$$

Employing Theorem 2 and the definition of the constant $c_{p}(\Omega)$ as maximal in the corresponding inequality, we obtain:

$$
\left(\frac{12}{p^{2} h(\Omega)}\right)^{\frac{p}{2}} \geqslant c_{p}(\Omega) \geqslant \frac{4^{p}}{p^{p} \gamma^{p}(\Omega)}
$$

Thus,

$$
\frac{\sqrt{3} \gamma(\Omega)}{2} \geqslant \sqrt{h(\Omega)}
$$

Combining this inequality with Osgood inequality [2, Ch. 3], [9],

$$
\gamma(\Omega) \leqslant \frac{2}{\alpha(\Omega)}
$$

we obtain the latter of the needed inequalities:

$$
\frac{\sqrt{3}}{\alpha(\Omega)} \geqslant \sqrt{h(\Omega)}
$$

This completes the proof. 


\section{EXAMPLES}

It is assumed in Theorem 3 that the parameters $p \in[1, \infty)$ ans $q \in[1, \infty)$ are fixed and satisfy the inequalities:

$$
\frac{1}{p}-\frac{1}{2} \leqslant \frac{1}{q} \leqslant \frac{1}{p}
$$

Hence, we obtain:

$$
\beta:=\frac{1}{q}-\frac{1}{p}+1 \in\left[\frac{1}{2}, 1\right] .
$$

Such choice of the restrictions for the parameters $p$ and $q$ is due to the fact that for a given domain, the inequality $h_{p, q}(\Omega)<\infty$ can hold not for all values of the parameters obeying the condition $1 \leqslant p \leqslant q<\infty$.

Let us show that $h_{p, q}(\Omega)=\infty$ for each simply-connected hyperbolic domain $\Omega$ under the condition $\beta \notin\left[\frac{1}{2}, 1\right]$. By the conformal invariance $h_{p, q}(\Omega)$, it is sufficient to consider the case, when $\Omega$ is some circle.

Example 1 Let $\Omega=\mathbb{D}=\{z \in \mathbb{C}:|z|<1\}$. We consider the circles

$$
\mathbb{D}_{r}=\{z \in \mathbb{C}:|z|<r\}
$$

of a radius $r \in(0,1)$. Since

$$
R(z, D)=1-|z|^{2}
$$

the hyperbolic area $V\left(\mathbb{D}_{r}\right)$ of the circle $\mathbb{D}_{r}$ and the hyperbolic length $A\left(\mathbb{D}_{r}\right)$ of the circumference $|z|=r$ are found explicitly. We have:

$$
V\left(\mathbb{D}_{r}\right)=4 \pi r^{2}\left(1-r^{2}\right)^{-1}
$$

and

$$
A\left(\mathbb{D}_{r}\right)=4 \pi r\left(1-r^{2}\right)^{-1}
$$

Therefore,

$$
\frac{V^{\beta}\left(\mathbb{D}_{r}\right)}{A\left(\mathbb{D}_{r}\right)}=(4 \pi)^{\beta-1} r^{2 \beta-1}\left(1-r^{2}\right)^{1-\beta} .
$$

If $\beta \notin\left[\frac{1}{2}, 1\right]$, then the considered quotient

$$
\frac{V^{\beta}\left(\mathbb{D}_{r}\right)}{A\left(\mathbb{D}_{r}\right)} \quad(0<r<1)
$$

is unbounded from above either in the vicinity of the point $r=0$ or in the vicinity of the point $r=1$. Thus,

$$
\sup _{r \in(0,1)} \frac{V^{\beta}\left(\mathbb{D}_{r}\right)}{A\left(\mathbb{D}_{r}\right)}=\infty
$$

under the assumption $\beta \notin\left[\frac{1}{2}, 1\right]$.

If $\Omega \subset \mathbb{C}$ is a domain with a uniformly perfect boundary, then $c_{p}(\Omega)>0$ for each $p \in[1, \infty)$. As it was mentioned above, for $p=2$ this fact was proved first by Fernández [5], while the general case is due to Avkhadiev [7]-[9]. If the boundary of the domain is not uniformly perfect, the issue on positivity of the constant $c_{p}(\Omega)$ becomes complicated. Namely, there exist domains $\Omega_{0}$ and $\Omega_{1}$, whose boundaries are not uniformly perfect, possessing the properties: $c_{p}\left(\Omega_{0}\right)>0$ and $c_{p}\left(\Omega_{1}\right)=0$. Appropriate examples of the domains $\Omega_{0}$ and $\Omega_{1}$ were given in [6]. For the completeness, we describe shortly these examples.

Example 2. Let

$$
\Omega_{0}=\mathbb{D} \backslash\left\{1-\frac{1}{2^{n}}\right\}_{n=1}^{\infty},
$$


where $\mathbb{D}=\{z \in \mathbb{C}:|z|<1\}[6]$. It is known that in the unit circle the hyperbolic distance $d_{\mathbb{D}}\left(z_{m}, z_{n}\right)$ between points $z_{m}, z_{n} \in \mathbb{D}$ is determined by the formula

$$
d_{\mathbb{D}}\left(z_{m}, z_{n}\right)=\frac{1}{2} \log \frac{1+t}{1-t}, \quad t=\left|\frac{z_{n}-z_{m}}{1-\bar{z}_{n} z_{m}}\right| .
$$

This is why the distance $d_{\mathbb{D}}\left(z_{m}, z_{n}\right)$ between the points $z_{m}=1-\frac{1}{2^{m}}$ and $z_{n}=1-\frac{1}{2^{n}}$ as $n \geqslant m+1$ is given by the formula

$$
\begin{aligned}
d_{\mathbb{D}}\left(z_{m}, z_{n}\right) & =\frac{1}{2} \log \frac{1-z_{n} z_{m}+z_{n}-z_{m}}{1-z_{n} z_{m}-z_{n}+z_{m}} \\
& =\frac{1}{2} \log \frac{1-\left(1-\frac{1}{2^{m}}\right)\left(1-\frac{1}{2^{n}}\right)-\frac{1}{2^{n}}+\frac{1}{2^{m}}}{1-\left(1-\frac{1}{2^{m}}\right)\left(1-\frac{1}{2^{n}}\right)+\frac{1}{2^{n}}-\frac{1}{2^{m}}}=\frac{1}{2} \log \frac{2^{n+1}-1}{2^{m+1}-1} .
\end{aligned}
$$

Therefore, we have

$$
\inf _{n \in \mathbb{N}, m \in \mathbb{N}, n \neq m} d_{\mathbb{D}}\left(z_{m}, z_{n}\right) \geqslant \frac{1}{2} \log 2>0 .
$$

Thanks to Corollary 2 we can state that for each $p \in[1, \infty)$, the constant $c_{p}\left(\Omega_{0}\right)$ is a positive number.

This example is interesting, while comparing it with the next example also considered in paper [6].

Example 3. Let

$$
\Omega_{1}=\mathbb{D} \backslash\{0\} \backslash\left\{\frac{1}{2^{n}}\right\}_{n=1}^{\infty},
$$

where $\mathbb{D}=\{z \in \mathbb{C}:|z|<1\}$.

The hyperbolic distance $d_{\mathbb{D}}\left(z_{m}, z_{n}\right)$ between the points $z_{m}=\frac{1}{2^{m}}$ and $z_{n}=\frac{1}{2^{n}}$ as $m \geqslant n+1$ is calculated explicitly by the formula:

$$
d_{\mathbb{D}}\left(z_{m}, z_{n}\right)=\frac{1}{2} \log \frac{1-z_{n} z_{m}+z_{n}-z_{m}}{1-z_{n} z_{m}-z_{n}+z_{m}}=\frac{1}{2} \log \frac{2^{m+m}+2^{m}-2^{n}+1}{2^{m+n}-2^{m}+2^{n}+1}, \quad n<m .
$$

As in the previous case, we have

$$
\inf _{n \in \mathbb{N}, m \in \mathbb{N}, n \neq m} d_{\mathbb{D}}\left(z_{m}, z_{n}\right) \geqslant \frac{1}{2} \log 2>0 .
$$

There is also a difference in comparison with the previous case; this is related with the point $0 \in A$. Since the distance between the points 0 and $z_{n}$ is given by the formula

$$
d_{\mathbb{D}}\left(0, z_{n}\right)=\frac{1}{2} \log \frac{1+z_{n}}{1-z_{n}}
$$

we shall obviously have $d_{\mathbb{D}}\left(0, z_{n}\right) \rightarrow 0$ as $n \rightarrow \infty$, i.e., the point $0 \in \mathbb{D}$ is an accumulation point of the sequence. Therefore,

$$
\inf _{z \in A, w \in A \backslash\{z\}} d_{\Omega}(z, w)=0, \quad A=\{0\} \cup\left\{\frac{1}{2^{n}}\right\}_{n=1}^{\infty} .
$$

In contrast to the previous case, we can not apply Corollary 2 to obtain inequality $c_{p}\left(\Omega_{1}\right)>0$. On the contrary, as it was shown in paper [6], the identity $c_{2}\left(\Omega_{1}\right)=0$ holds. Employing Theorem 1, we obtain that $c_{p}\left(\Omega_{1}\right)=0$ for all $p \in[1,2)$. 


\section{BIBLIOGRAPHY}

1. L.V. Ahlfors. Conformal invariants, Topics in Geometric Function Theory. McGraw-Hill, New York (1973).

2. F.G. Avkhadiev, K.-J. Wirths. Schwarz-Pick type inequalities. Birkhäuser Verlag, Basel (2009).

3. Ch. Pommerenke. Uniformly perfect sets and the Poincaré metric // Arch. Math. 32:1, 192-199 (1979).

4. D. Sullivan. Related aspects of positivity in Riemannian geometry // J. Diff. Geom. 25:3, 327-351 (1987).

5. J.L. Fernández. Domains with strong barrier // Revista Matemática Iberoamericana. 5:2, 47-65 (1989).

6. J.L. Fernández, J.M. Rodríguez. The exponent of convergence of Riemann surfaces, bass Riemann surfaces // Ann. Acad. Sci. Fenn. Series A. I. Mathematica. 15, 165-183 (1990).

7. F.G. Avkhadiev. Hardy type inequalities in higher dimensions with explicit estimate of constants // Lobachevskii J. Math. 21, 3-31 (2006).

8. F. G. Avkhadiev. Hardy-type inequalities on planar and spatial open sets // Trudy Matem. Inst. Steklov. 255, 8-18 (2006). [Proc. Steklov Inst. Math. 255, 2-12 (2006).]

9. F.G. Avkhadiev. Integral inequalities in domains of hyperbolic type and their applications // Matem. Sborn. 206:12, 3-28 (2015). [Sb. Math. 206:12, 1657-1681 (2015).]

10. F.G. Avkhadiev, R.G. Nasibullin, I.K. Shafigullin. $L_{p}$-versions of one conformally invariant inequality // Izv. VUZov. Matem. 8, 88-92 (2018). [Russian Math. (Iz. VUZ). 62:8, 76-79 (2018).]

11. M. Hoffmann-Ostenhof, T. Hoffmann-Ostenhof, A. Laptev. A geometrical version of Hardy's inequality // J. Funct. Anal. 189:2, 539-548 (2002).

12. F.G. Avkhadiev, K.-J. Wirths. Unified Poincaré and Hardy inequalities with sharp constants for convex domains // Z. Angew. Math. Mech. 87:8-9, 632-642 (2007).

13. F.G. Avkhadiev, K.-J. Wirths. Weighted Hardy inequalities with sharp constants // Lobachevskii J. Math. 31:1, 1-7 (2010).

14. F.G. Avkhadiev, K.-J. Wirths. Sharp Hardy-type inequalities with Lamb's constants // Bull. Belg. Math. Soc. Simon Stevin. 18:4, 723-736 (2011).

15. F.G. Avkhadiev,K.-J. Wirths. On the best constants for the Brezis-Marcus inequalities in balls // J. Math. Anal. Appl. 396:2, 473-480 (2012).

16. F.G. Avkhadiev, I.K. Shafigullin. Sharp estimates of Hardy constants for domains with special boundary properties // Izv. VUZov. Matem. 2, 69-73 (2014). [Russ. Math. (Iz. VUZ). 58:2, 58-61 (2014).]

17. F.G. Avkhadiev, R.G. Nasibullin. Hardy-type inequalities in arbitrary domains with finite inner radius // Sibir. Matem. Zhurn. 55:2, 191-200 (2014). [Siberian Math. J. 55:2, 191-200 (2014).]

18. A.A. Balinsky, W.D. Evans, R.T. Lewis. The analysis and geometry of Hardy's inequality. Springer, Heidelberg (2015).

19. R.G. Nasibullin. Sharp Hardy type inequalities with weights depending on Bessel function // Ufimskij Matem. Zhurn. 9:1, 89-97 (2017). [Ufa Math. J. 9:1, 89-97 (2017).]

20. I.K. Shafigullin. Lower bound for the Hardy constant for an arbitrary domain in $\mathbb{R}^{n} / /$ Ufimskij Matem. Zhurn. 9:2, 104-111 (2017). [Ufa Math. J. 9:2, 102-108 (2017).]

21. S.L. Sobolev. Some applications of functional analysis in mathematical physics. Nauka, Moscow (1988). [Transl. Math. Monog. 90. Amer. Math. Soc. Providence, RI (1991).]

22. V.G. Maz'ya. Sobolev Spaces. Springer-Verlag, Berlin (1985).

23. V.M. Miklyukov, M.K. Vuorinen Hardy's inequality for $W_{0}^{1, p}$-functions on Riemanni an manifolds // Proc. Amer. Math. Soc. 127:9, 2745-2754 (1999).

24. V. Alvarez, D. Pestana, J.M. Rodríguez. Isoperimetric inequalities in Riemann surfaces of infinite type // Revista Matemática Iberoamericana. 15:2, 353-425 (1999). 
Farit Gabidinovich Avkhadiev,

Kazan Federal University,

Kremlevskaya str., 18,

420008, Kazan, Russia

E-mail: avkhadiev47@mail.ru

Ramil Gaisaevich Nasibullin,

Kazan Federal University,

Lobachevsky Institute of Mathematics and Mechanics

Kremlevskaya str., 18,

420008, Kazan, Russia

E-mail: NasibullinRamil@gmail.com

Ilnar Kasyjmovich Shafigullin,

Lobachevsky Institute of Mathematics and Mechanics

Kazan Federal University,

Kremlevskaya str., 18,

420008, Kazan, Russia

E-mail: shafigullin.ik@gmail.com 